\title{
ERRATUM
}

\section{Photoperiodic regulation of cellular retinoic acid-binding protein 1, GPR50 and nestin in tanycytes of the third ventricle ependymal layer of the Siberian hamster}

\author{
Perry Barrett, Elena Ivanova, E Scott Graham, Alexander W Ross, Dana Wilson, Helene Plé, \\ Julian G Mercer, Francis J Ebling, Sandrine Schuhler, Sandrine M Dupré, Andrew Loudon \\ and Peter J Morgan
}

The authors and the journal apologise for the incorrect definition of the gene, CRBP1 in this article that was published in 2006, 191, 687-698.

The incorrect expansion of the abbreviation appears in the title and abstract (page 687), materials and methods (page 689), and the heading of the first results subsection (page 690), which should all read cellular retinol binding protein, CRBP1 and not as published. 